\title{
THE HITTING CHARACTERISTICS OF A STRONG MARKOV PROCESS, WITH APPLICATIONS TO CONTINUOUS MARTINGALES IN $R^{n}$
}

\author{
BY G. E. DENZEL
}

Communicated by M. Loeve, July 11, 1966

1. Introduction. M. Arbib showed in [1] that, essentially, on the real line a continuous path process with the same "hitting characteristics" as a diffusion was itself a diffusion (strong Markov process). His methods did not lend themselves to more general processes. The purpose of this note is to give a general characterization along this line, for right continuous, nonterminating, quasi-left continuous strong Markov processes with left limits, taking their values in a locally compact, noncompact second countable space $E$. We also give some interesting consequences concerning continuous martingales in $R^{n}$. Full proofs of these and related results will appear elsewhere.

2. Hitting characteristics. Let $\hat{X}$ be a process as above, described by measures $\hat{P}^{x}(x \in E)$ on the space of paths (assume that the function $x \rightarrow \hat{P}^{x}(A)$ is Borel measurable for all Borel $A \subset E$, and that $\left.\hat{P}^{x}\left(X_{0}=x\right)=1\right)$. Let $F_{t}$ be the $\sigma$-field generated by the path functions $X_{s}(s \leqq t)$, and let $F_{R}^{+}$be the $\sigma$-field of the stopping time $R\left(A \in F_{R}^{+}\right.$ if $A \cap\{R<t\} \in F_{t}$ for all $t$ ). Another process $X$, described by a measure $P$ on the same path space, will be said to have the same hitting characteristics as $\hat{X}$ if

$$
\begin{aligned}
E\left[T_{\bar{G}} c \circ \theta_{R} \mid F_{R}^{+}\right] & =\hat{E}^{X_{R}}\left[T_{\bar{G}} c\right] \quad \text { P-a.s., } \\
E\left[I_{B} \circ X_{T_{\bar{G}^{c}}} \circ \theta_{R} \mid F_{R}^{+}\right] & =E^{\mathrm{X}_{R}}\left[I_{B} \circ X_{T_{\bar{G}^{c}}}\right] \quad \text { P-a.s. }
\end{aligned}
$$

for every stopping time $R$, Borel set $B \subset E$, and open set $G \subset E$ with compact closure ( $T \bar{G}_{n}^{c}$ is the first hitting time of the complement of the closure of $G$, and $\theta_{R}$ is the shift by $R$ ).

We write $\mu$ for the distribution of $X_{0}$ under $P$.

THEOREM 1. If $X$ and $\hat{X}$ have the same hitting characteristics as described above, and if there is a sequence of sets $G_{n} \nearrow E, G_{n}$ open with compact closure, such that $x \rightarrow \hat{E}^{X}\left[T_{\bar{G}_{n}^{c}}\right]$ is a bounded function on $E$, then $P=\hat{P}^{\mu}-i . e ., X$ is a strong Markovprocess.

The existence of the sets $G_{n}$ follows whenever $\hat{X}$ is, say, a Feller 
process. ${ }^{1}$ The proof of Theorem 1 is by standard techniques from the theory of Markov process.

3. Consequences. Arbib used his theorem to generalize Levy's martingale characterization of Brownian motion [2] to other diffusions on the real line. We similarly use ours to obtain the following theorem.

Theorem 2. If $\left(X_{t}\right)$ is a process in $R^{n}$ such that $\left(h \circ X_{t}\right)$ is a continuous local martingale for every spherical harmonic polynomial $h$, and if $\left|X_{t}\right|^{2} / n-t$ is a continuous local martingale, then $\left(X_{t}\right)$ is a Brownian motion.

Dambis [3] proved that on the line any continuous martingale $\left(X_{t}\right)$ with $X_{0}=0$ was equivalent to a continuous random time change of Brownian motion. Using a slight generalization of his methods, and our Theorem 2, we obtain the following result.

Theorem 3. If $\left(X_{t}\right)$ is a process in $R^{n}$ with $h \circ X_{t}$ a continuous local martingale for every spherical harmonic polynomial $h$, and if $X_{0}=0$, then $\left(X_{t}\right)$ is equivalent to a continuous random time change of Brownian motion.

A slightly weaker version of this last result was obtained by Dubins and Schwarz [4] and by Kunita and Watanabe [5], both using much different techniques. The latter paper also contains a different proof of Theorem 2 .

\section{REFERENCES}

1. M. Arbib, Hitting and martingale characterizations of one-dimensional diffusions, Z. Wahrsch. 3 (1965), 232-247.

2. P. Lèvy, Processus stochastiques et mouvement Brownien, Gauthier-Villars, Paris, 1948, p. 78.

3. K. E. Dambis, On the decomposition of continuous submartingales, Teor. Verojatnost. i Primenen. 10 (1965), 438-448. (Russian)

4. L. E. Dubins and Gideon Schwarz, On continuous martingales, Proc. Nat. Acad. Sci. 53 (1965), 913-916.

5. Hiroshi Kunita and Shinzo Watanabe, On square integrable martingales, Privately communicated preprint, 1966.

\section{Dartmouth College}

1 (On a space $E$ such that $\hat{P}^{x}\left[T_{K^{c}}<\infty\right]>0 \forall x \in E$, compact $K \subset E$. This might be called the "natural" state space.) 